\title{
Diode-Pumped Mode-Locked LiSAF Laser
}

Final Report

Contract no DE-FG03-92ER81291

February 1992 to February 1996

Light wave alectronics Corporation

1161: an Antonio Road

Mountain View, CA 94043

(415) 962-0755

February 1996 


\section{DISCLAIMER}

This report was prepared as an account of work sponsored by an agency of the United States Government. Neither the United States Government nor any agency thereof, nor any of their employees, make any warranty, express or implied, or assumes any legal liability or responsibility for the accuracy, completeness, or usefulness of any information, apparatus, product, or process disclosed, or represents that its use would not infringe privately owned rights. Reference herein to any specific commercial product, process, or service by trade name, trademark, manufacturer, or otherwise does not necessarily constitute or imply its endorsement, recommendation, or favoring by the United States Government or any agency thereof. The views and opinions of authors expressed herein do not necessarily state or reflect those of the United States Government or any agency thereof. 


\section{DISCLAIMER}

Portions of this document may be illegible in electronic image products. Images are produced from the best available original document. 


\section{Table of Contents}

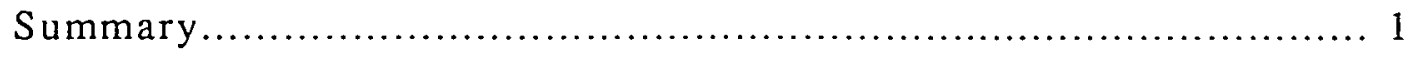

1. Background and Motivation.................................................. 4

2. Laboratory Results........................................................ 8

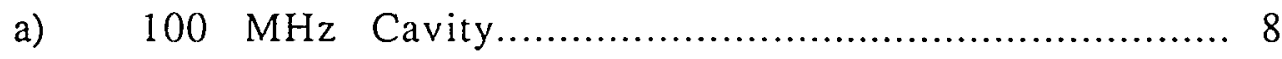

b) $\quad 500 \mathrm{MHz}$ Cavity .............................................. 10

c) $532 \mathrm{~nm}$ pumping results....................................... 11

3. Improved Diode Pumping............................................ 13

4. New Diode based Solid State Pump Source..................................... 14

a) System Design and Optical challenges.................................. 16

b) Mechanical Mounting.............................................. 22

c) Electronics and Control Module........................................ 24

5. Conclusion and Future Directions............................................ 25






\section{LEGHTWAVE}

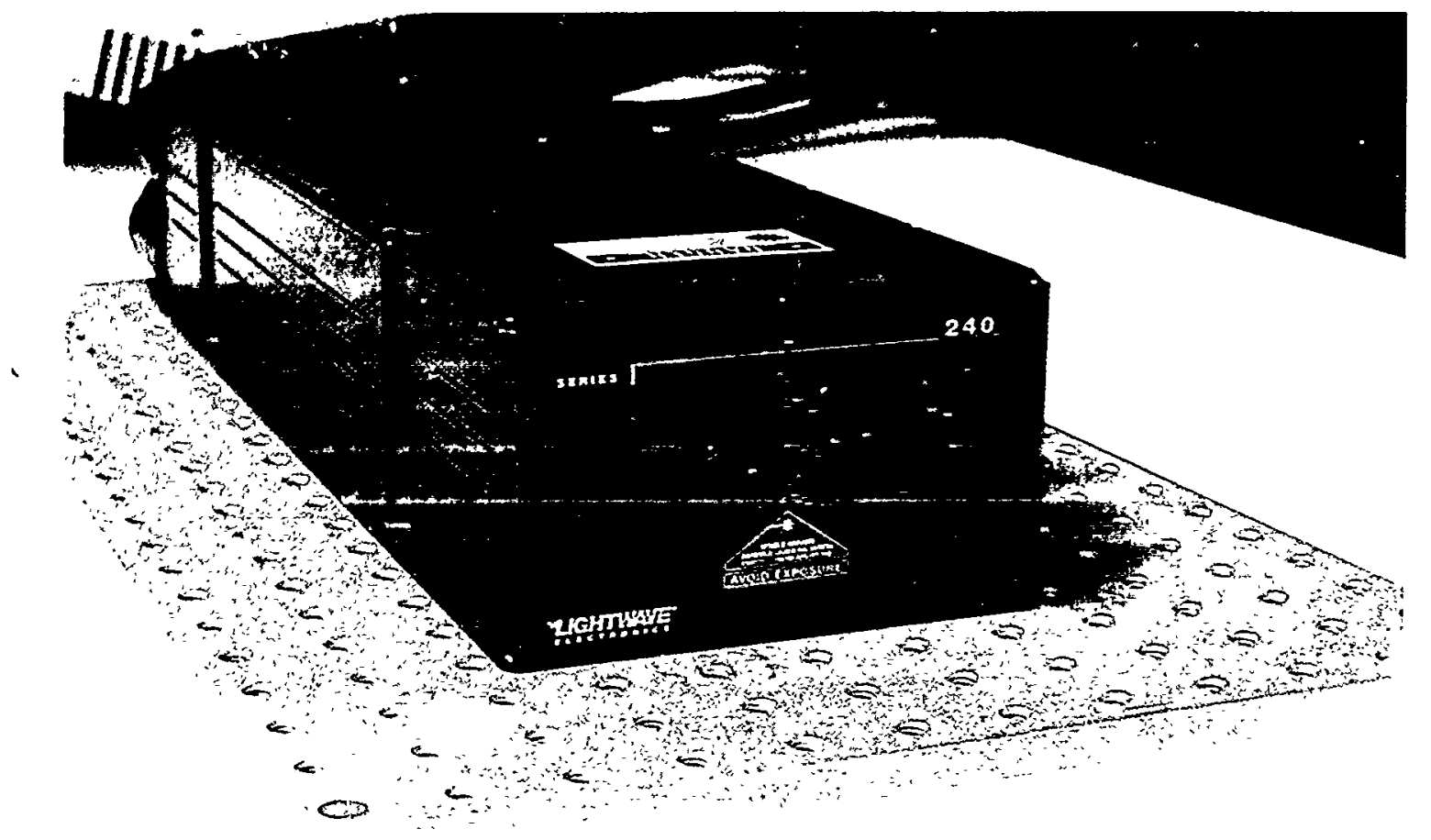




\section{Summary}

Under this contract we have developed $\mathrm{Cr}^{3+}$ LiSrAlF$_{6}(\mathrm{Cr}: \mathrm{LiSAF}, \mathrm{LiSAF})$ modelocked lasers suitable for generation of polarized electrons for CEBAF. As $670 \mathrm{~nm}$ is an excellent wavelength for optical pumping of $\mathrm{Cr}: \mathrm{LiSAF}$, we have used a LIGHTWAVE developed $670 \mathrm{~nm}$ diode pump module that combines the output of ten diode lasers and I: s a rproximately 2 Watts of optical power. Ry the use of a Niffraction limited pump beam however, it is possible to maintain a small mode size through the length of the crystal and hence extract more power from Cr:LiSAF laser. For this purpose we have developed a 1 Watt, red 660nm laser (LIGHTWAVE model 240R) which serves as an ideal pump for Cr:LiSAF and is a potential replacement of costly and less robust krypton laser. This new system is to compliment LIGHTWAVE Series 240 , and is currently being considered for commercialization. Partially developed under this contract is LIGHTWAVEs product model 240 which has already been in our production lines for a few months and is commercially available. This laser produces 2 Watts of output at $532 \mathrm{~nm}$ using some of the same technology developed for production of the $660 \mathrm{~nm}$ red system. It is a potential replacement for argon ion lasers and has better current and cooling requirements and is an excellent pump source for $\mathrm{Ti}: \mathrm{Al}_{2} \mathrm{O}_{3}$. Also, as a direct result of this contract we now have the capability of commercially developing a mode-locked $100 \mathrm{MHz} \mathrm{Cr}: \mathrm{LiSAF}$ system. Such a laser could be added to our $100 \mathrm{MHz}$ LIGHTWAVE Series 131. The Series 131 lasers provide pico second pulses and were originally developed under another DOE SBIR. Both models of LIGHTWAVE Series 240 lasers, the fiber coupled pump module and the $100 \mathrm{MHz}$ LiSAF laser of Series 131 have been partially developed under this contract, and are commercially competitive products. 


\section{Background and Motivation}

Continuous Electron Beam Facility (CEBAF) currently uses a CW Ti:sapphire laser to produce polarized electrons. Unfortunately this system results in degradation of the photocathode at a rate 6 times higher than would occur if a suitable pulsed laser were used. Development of an all solid state tunable laser suitable for generating polarized electrons for CEBAF could improve overall system performance considerably with and in phase II of this project we proposed to develop such a laser.

A number of Department of Energy :rograms require a stable, efficient, shortpulsed laser operating at high repetition frequencies. One application is to generate short pulses of electrons on photo-cathodes for plasma accelerators and free electron lasers [1,2]. Another application is beat wave accelerator experiments [2]. Short pulse lasers with low timing jitter also would find use for precise switching of power linacs [2,3]. Modelocked lasers are in demand for many applications. One application is optical projing of integrated circuits, such as electro-optic sampling [3,4] or charge sensing in silicon ICs [1]. Medical applications such as precision eye surgery are possible [4]. This laser could provide the seed pulse for an amplified system to produce tera Watt peak powers [4], for applications such as X-ray generation and study of non-linear optical phenomena. In general, LIGHTWAVE's main goal in designing laser systems is to create easy to use and hands-off technology that would find instrumentation and industrial applications.

Solid siate lasers pumped by diode lasers offer many desired features not provided by conventional flashlamp-pumped solid state systems $[1,2]$. The good spectral match of the diode output to the absorption of the solid state laser materials provides for high overall optical efficiency, and extra pump power that must be removed as waste heat from the crystal is reduced. Also, the projected lifetime of diode lasers is $>10,000$ hours [1], far surpassing the typical flashlamp lifetime of several hundred hours. 
One of our goals was to get short pulse widths directly from the laser. The pulse width in a mode-locked laser is predicted by Kuizenga-Siegman theory which states that ihe pulsewidth $\tau_{m l}$ is given by

$$
\tau_{m l}=0.45\left(\frac{g_{0}}{\Delta_{m}}\right)^{1 / 4} \sqrt{\frac{1}{f_{m} f_{a}}}
$$

where $g_{G}$ is the round trip saturated gain of the laser, $\Delta_{m}$ is the depth of modulation introduced by the mode-locker, $f_{m}$ is the modulation frequency, and $f_{a}$ is the gainbandwidth (typically assumed to equal the fluorescence linewidth) of the optical transition. Increasing the laser material's gain-bandwidth allows for shorter pulse widths.

Decreasing the pulse width is also possible by increasing the laser's pulse repetition rate $f_{m}$, which is done by decreasing the laser cavity size. The laser's pulse repetition rate is the inverse of the cavity round trip time, i.e. $f_{m}=c / 2 L$, where $L$ is the length of the cavity and $c$ is the free-space speed of light. Increasing the pulse rate from $76 \mathrm{MHz}$ to 250 $\mathrm{MHz}$ should decrease the pulse width by about $50 \%$. Another motivation to increase the pulse rate is to decrease the mode-locked pulse build-up time, also given by KuizengaSiegman theory to be

$$
T_{s s}=\frac{f_{a}}{f_{m}^{2}} \sqrt{\frac{1}{8 g_{0} \Delta_{m}}}
$$

Shorter pulse build-up times lead to more stable, robust lasers that are less sensitive to mechanical vibrations and disturbances.

Improving the mode locker performance, i.e., improving its diffraction efficiency, is a third way to decrease mode-locked laser pulse width. The mode locker is an optical modulator, typically an acousto-optic device, that modulates the light at the laser pulse rate. Typical mode lockers are made from a fused quartz substrate and have a lithium niobate 
transducer thinned to about one-half an acoustic wavelength. Driving the transducer with an electrical signal generates an acoustic wave through the piezo-electric effect. The acoustic wave couples into the substrate, where it reflects off the back surface to form an acoustic standing wave. The acoustic wave causes a periodic variation in the index of refraction in the fused quartz, which scatters or diffracts an optical beam passing through this region. This technology has several potential technical challenges at higher drive frequency. Acoustic loss, which increases as the square of the frequency, starts to become cionificant at drive frequencies greater than $100 \mathrm{M} \mathrm{Hz}_{\mathrm{z}}$. If the acoustic beam is significantly attenuated, reduced diffraction efficiency may result. Also, the lithium niobate transducer becomes much harder to fabricate at higher frequencies because of the reduced thickness required. Finally, there is a trade-off between the diffraction efficiency, which is proportional to the length, and the sensitivity of the diffraction to the angle between the optical beam and the acoustic waves (known as the Bragg angle), which increases as the square of the drive frequency.

\section{Cavity Design, Experimental Techniques and Challenges}

Figure 1 shows the cavity used for our picosecond modelocked lasers. We call this an asy-nmetric V-cavity. It starts with the crystal flat surface high reflection coated for ope ation at $850 \mathrm{~nm}$, which forms one end of the cavity. The other Brewster-angled surface serves as a low loss, broadband and simple intracavity surface. The curved mirror focuses the laser mode to a small spot size in the crystal. In order to increase laser gain, the spot size must be as small as possible. Of course a spot size that is too small could result in damage to the crystal especially since high doping levels are possible. Also the reflective coating can be damaged effecting overall laser performance. The spot size must also be equal to or higher than the cavity mode size to ensure mode matching for efficient operation

and clean spacial mode performance. The laser mode size in the long leg of the cavity is nearly collimated and allows for easy insertion of an intracavity modulator as depicted in 




Figurre 1 
the figure. This cavity was chosen with commercialization of modelocked lasers in mind. The ability to use existing technology currently in our production lines for manufacturing LIGHTWAVE model 131, 100 MHz YAG or YLF modelocked lașers, allowed a more timely and efficient product development without having to re-engineer many mechanical and optical parts. This way we minimized the number of parts that needed to be modified and designed a system with features which many of our customers are already familiar with and demand.

Since the modelocker diffraction efficie...y is reduced with in :sasing frequency, it becomes much more difficult to obtain lasing action as the cavity size is reduced. Experimental techniques allowed us to overcome many difficulties associated with modelocked operation $500 \mathrm{MHz}$. As with any modelocked laser, cavity length must match the optical modulation frequency. If the diffraction efficiency of the modulator is high, this task can be achieved by maintaining a constant RF signal to the modulator and changing the cavity length to match the oscillation frequency. Once a good match is obtained modelocked lasing action will take place. For operation at $500 \mathrm{MHz}$ however, this simple procedure was not enough

In order to obtain the maximum amount of diffraction efficiency from a modulator, the modelocker temperature was observed closely. By observation of the RF power reflected from the modulator as a function of modelocker temperature, the optimal moduli $r$ temperature can be determined at a given drive frequency. As the crystal temperatur. increased, depth of modulation $\left(\Delta_{\mathrm{m}}\right.$.in equation 1.1) changes. At the optimal temperature, the accoustic waves inside the modulator best match operation at the drive frequency and the amount of reflected RF is reduced. It is then imperative to maintain this temperature for best performance. In the case for the $500 \mathrm{MHz}$ LiSAF cavity, the temperature window where lasing action would take place was about $1 / 2^{\circ} \mathrm{C}$. In this case, the diffraction efficiency of the optical modulator was so low that the above two procedures still did not produce modelocked lasing action. The next step was to introduce small amplitude and 
random vibrations to the modelocker. These tapping vibrations move the diffracted beam and allow unstable and temporary lasing action to take place. By observing the frequency of the output light on a spectrum analyzer, and changing the RF frequency, it was possible to match the cavity and optical modulation frequencies. But since the RF frequency was then changed, it was necessary to scan the modelocker temperature again to obtain maximum diffraction efficiency. Repeating the above procedure several times, enabled us to obtain modelocked lasing action with good pulse stability (performance data is given in Laboratory Results section)

There were also many technical challenges relating to quenching of the upper state lifetime of Cr:LiSAF. Since the upper state lifetime of the Cr:LiSAF drops with temperature it is important to maintain the crystal at relatively low temperatures. To ensure a relatively long lifetime $(65 \mu \mathrm{s})$ we decreased the crystal temperature to $10^{\circ} \mathrm{C}$. Cooling the crystal was achieved by the use of a closed loop controlled thermoelectric cooler (TEC). The TEC was mounted to a water cooled aluminum block. This of course meant that we not only had to have a water reservoir and a water-air heat exchanger, but also a water pump and an electric motor. To minimize the amount of noise introduced to our laser from the water pump and the electric motor we used about 6 meters of $1 / 8$ " flexible vinyl tubing and kept the cooling support system as far away from the optical components as we could. Another technical challenge was keeping optically active surfaces clean and clear.Condensation forming on crystal surfaces would not only attract dust and increase chances of burns but also effect the performance of the coatings and potentially damage surface quality permanently. To avoid these problems we used a plastic housing placed around the crystal and flowed medical quality dry air through it. This of course meant that we needed to have a compressed air reservoir and pressure regulating gauges and a flow controller. Fortunately we were able to obtain needed materials and address the above -issues properly. 


\section{Iaboratory Results}

\section{a) 100 MHz Cavity}

In this project we obtained $100 \mathrm{MHz}$ modelocked lasing action using a $1.5 \% \mathrm{Cr}$ doped LiSAF crystal. Figure 2 shows an oscilloscope trace of a pulse as detected by a fast photodetector (Antel model AR-S2). The pulse response of the photo detector was about $70 \mathrm{ps}$ (FWHM) and its responsivity was $95 \%$ at $850 \mathrm{~nm}$ with a rise time of $<35 \mathrm{ps}$. To make more accurate pulsewidth measurements, we used a LIGHTWAVE manufactured

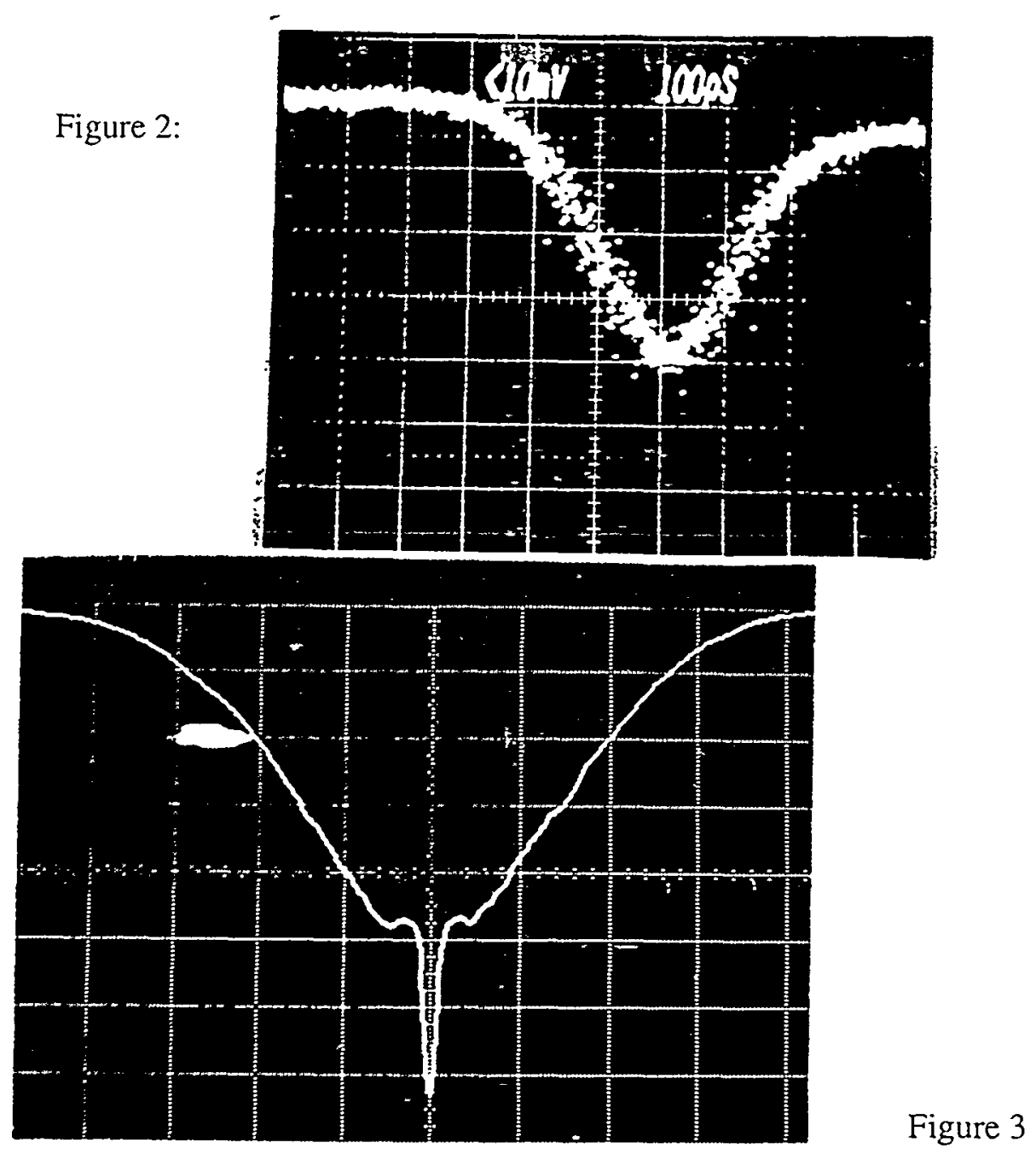

autocorrelator. Figure 3 is an oscilloscope trace of the autocorrelator signal. We obtained $90 \mathrm{ps}$ pulses at $640 \mathrm{~mW}$ of pump power with a $2 \%$ output coupler. As depicted by this figure, the pulse FWHM is about $90 \mathrm{ps}$. During these measurements the modelocker was kept at its resonance temperature of $55.6^{\circ} \mathrm{C}$, and the reflected RF was closely watched to 
ensure that the modelocker was operating at its maximum efficiency. Figure 4 is a plot of output vs. input powers. We observed a threshold power of $510 \mathrm{~mW}$ with an 80 micron waist in the crystal and obtained a slope efficiency of $15.4 \%$.

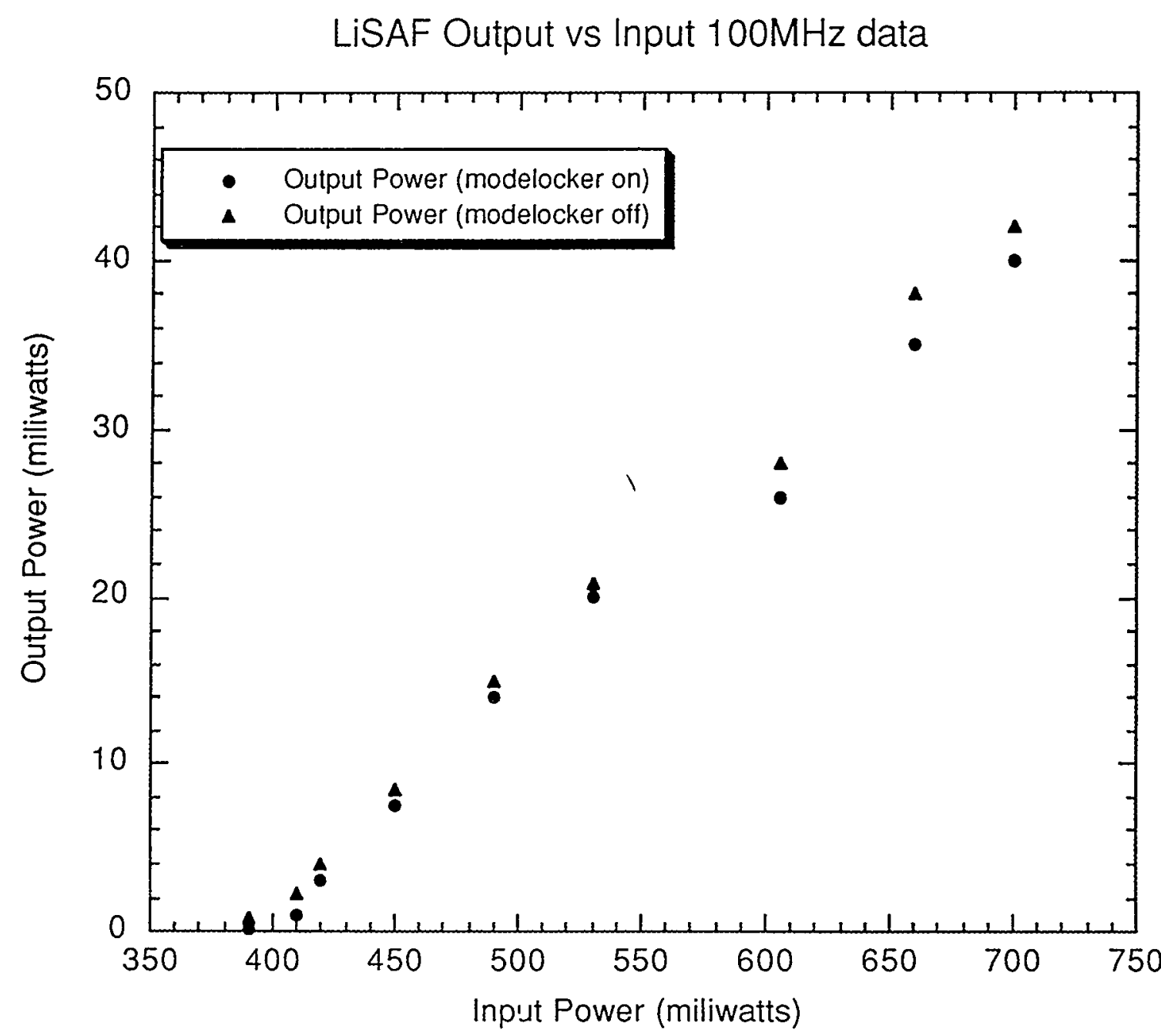

Figure 4

An ambient temperature change would cause the mirrors and optical mounts to move and result the laser cavity to shift out of resonance with the optical drive frequency. Our laser seemed quite robust and would run continuously modelocked for a period of hours without -moving out of resonance. 


\section{b) $500 \mathrm{MH} \%$ Cavity}

At this higher frequency, we observed improved power and pulse performance. As distances between optical components were shorter, the laser was quite stable. Figure 5 shows a plot of laser output vs. input powers. We obtained our shortest pulses of $66 \mathrm{ps}$ at an average power of $54 \mathrm{~mW}$ at a pump power of $640 \mathrm{~mW}$. We maintained the same 80 micron waist in the crystal and observed a threshold pump power of $424 \mathrm{~mW}$.

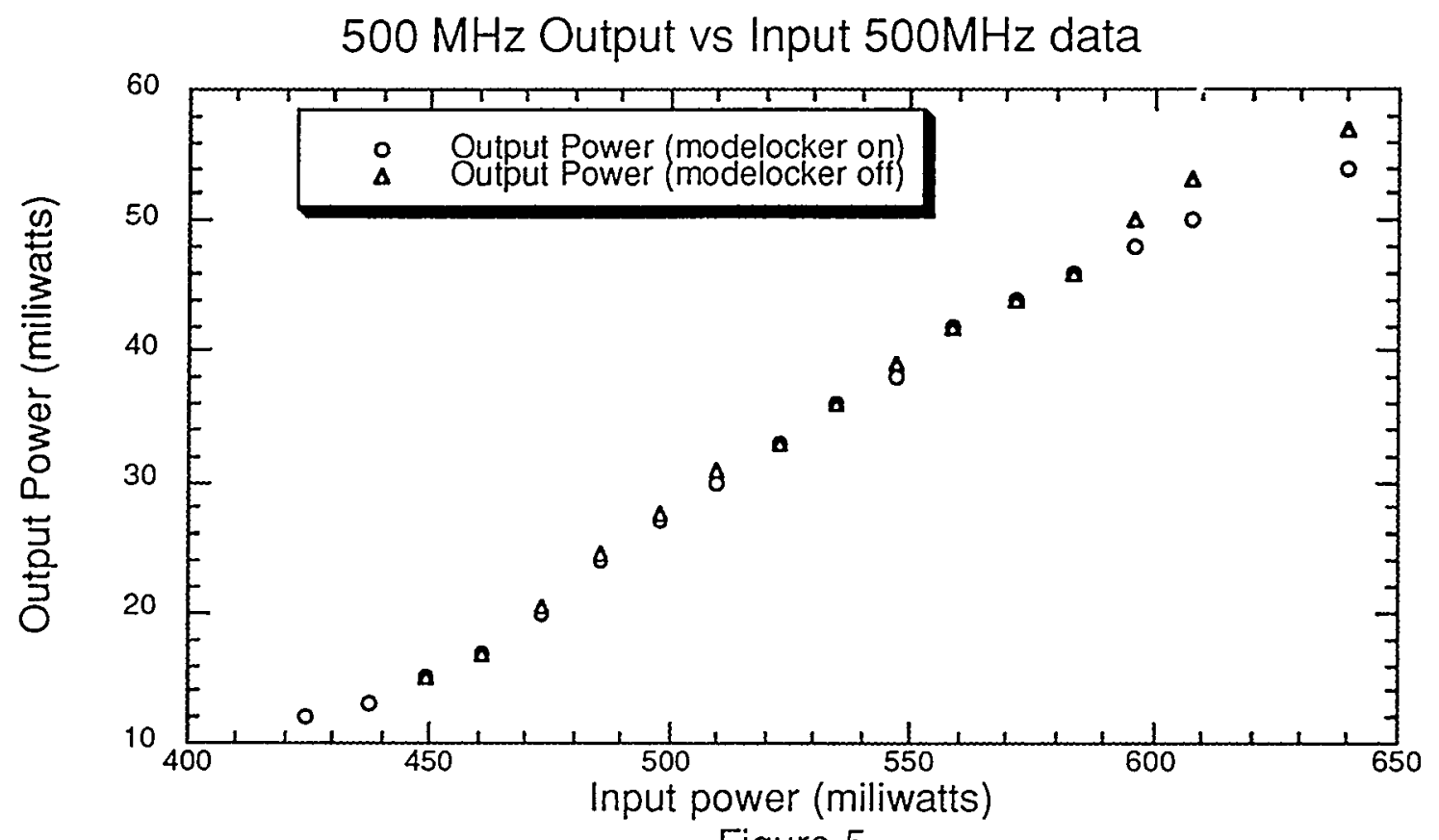

Figure 5

We observed a slope efficiency of $22.1 \%$. The LiSAF crystal was cooled to $5.2^{\circ} \mathrm{C}$. We also made rough wavelength measurements and observed lasing at $836-844 \mathrm{~nm}$. There were no tuning elements in the cavity. Once again we used a .2\% output coupler. For this optical modulation frequency we obtained modelocker resonance at $54.0^{\circ} \mathrm{C}$ and were able to maintain lasing action for several hours without a need to readjust components. We also looked at the output pulse frequency with a spectrum analyzer (HP model \# 8591A). Figure 6 shows a spectrum analyzer trace. We observed our sidebands to be about $35 \mathrm{KHz}$ 


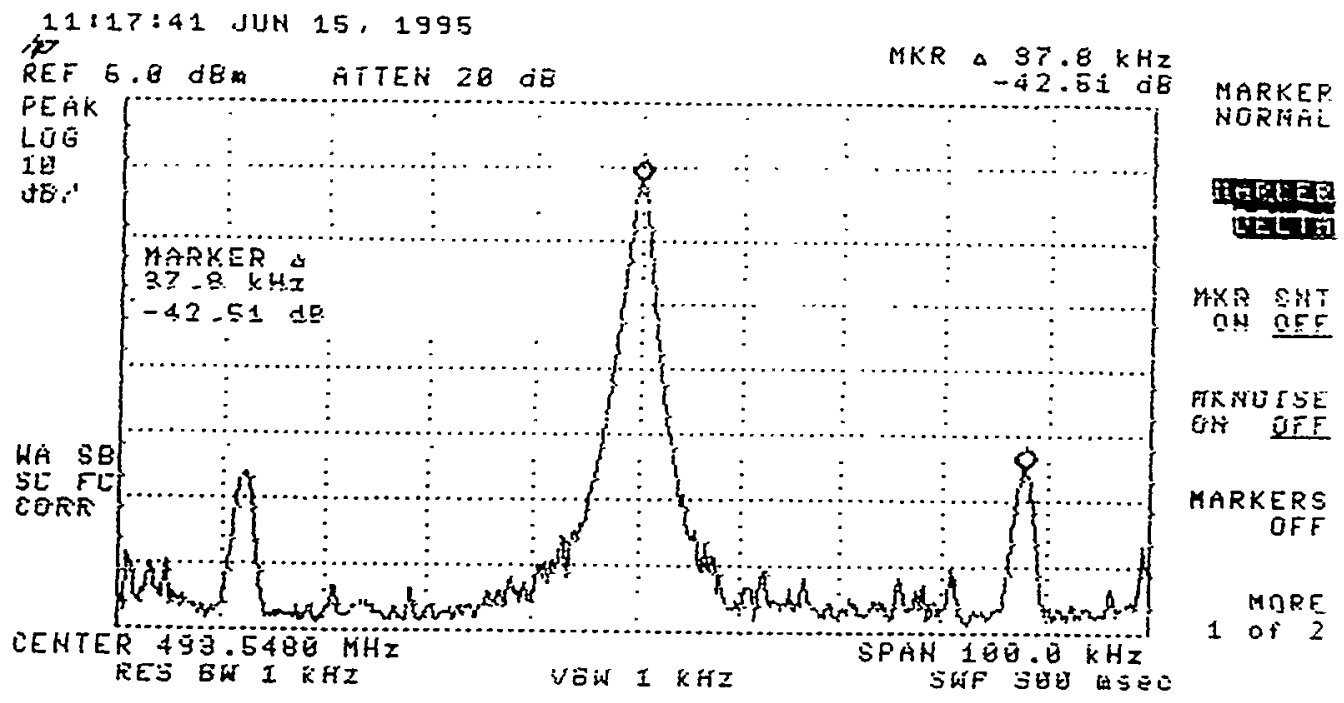

Figure 6

away from the peak frequency and about $40 \mathrm{~dB}$ lower in amplitude. FWHM of the modelocked frequency response was about $7 \mathrm{kHz}$, and the sideband amplitudes fell $44 \mathrm{~dB}$ below the peak amplitude; providing a very quiet system. The sidebands are result of relaxation oscillations, they contain most of the laser's amplitude noise.

\section{c) 532nm Pumping results.}

Since the absorption of the Cr:LiSAF is broad band and high doping levels are possible, it is possible t. pump the material with wavelengths well below its peak absorption region. In thi. experiment we used a diode pumped solid state frequency doubled YAG laser to pump a $6.5 \%$ LiSAF crystal at $532 \mathrm{~nm}$. Figure7 shows the schematic of a simple cw cavity used. Here we used a $.17 \%, 10 \mathrm{~cm}$ concave output

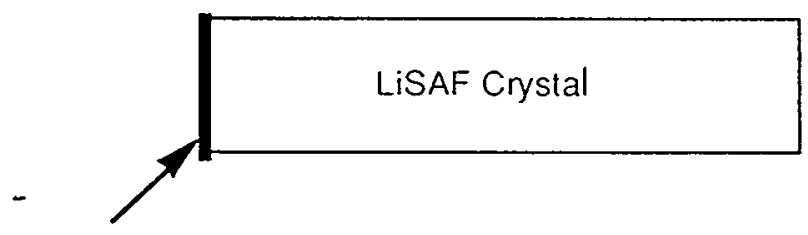

High reflective surface@850nm
Figure 7

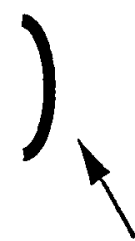

$99.97 \%$ reflective mirror, $\mathrm{RoC}=10 \mathrm{~cm}$ 




Figure 8

coupler. We matched the pump beam with the $90 \mu$ cavity mode size and used the reflection coating on one side of the crystal to serve as a second mirror.

With this configuration we obtained $39 \mathrm{~mW}$ o: $R$ power at a pump power of 1.10 watts. Once again the crystal was cooled and maini, , , at $10^{\circ} \mathrm{C}$. Figure 8 shows a plot of LiSAF output power vs $532 \mathrm{~nm}$ pump power. As t.e pump power is increased thermal effects become more significant and reduce performance of the laser. We observed a laser threshold of $232 \mathrm{~mW}$ and a slope efficiency of $2.6 \%$ before thermal effects were noticeable. We observed thermal effects at approximately 1.8 watts of pump power. Through this experiment we have shown that it is possible to obtain LiSAF lasing action with the use of one of our newly developed $532 \mathrm{~nm}$ LIGHTWAVE Model 240 lasers. 


\section{Improved Diode Pumping}

The pump module is a device developed by LIGHTWAVE for generating a high power bright pump beam. The device is shown in Figure 9. This system is not commercially available. However we had access to one unit in the laboratory, which we used for our experiments. The pump module multiplexes the output of five $.5 \mathrm{~W}, 500 \mu \mathrm{m}$ stripe laser diodes into a $100 \mu \mathrm{m}$ diameter fiber with a numerical aperture (NA) of 25 . Spacial mixing in the fiber leads to a uniform circular spot.



Figure 9

The module itself is air cooled, and along with its driver is housed in a box with dimensions of $6 \times 18 \times 18$. There are two multiplexed diode mechanisms in this pump module. This would allow end pumping of an active medium from both sides using only one module. For the purposes of this project we used one port of the $670 \mathrm{~nm}$ pump module which delivered $700 \mathrm{~mW}$ from a $100 \mu \mathrm{m}$ diameter fiber. 
This device is very user friendly and extremely simple to use. On its front panel, it has an on/off button and two 100 turn potentiometers. Each potentiometer is assigned to control the output power from a specific fiber port. This way the user is able to control the amount of pump light focused into the active medium with minimal effort. The device uses a standard $120 \mathrm{~V}, 60 \mathrm{~Hz}$ power supply; and since it is air cooled it is easily transportable. The electronic controls allow for a complete disabling of either port just in case the user does not wish to utiliza the ports for an extended period of time.

Although this module is an exceller.t pump source for Cr:Lis.:. many applications call for a diffraction limited pump beam.A diffraction limited source allows more efficient pumping. With such a source the effective length of an active medium can be maximized resulting in better extraction of energy, higher optical efficiency, and better spacial beam quality. Since the pump module uses 5 laser diodes, the total cost of the system is high. This in itself makes this product less attractive to those who wish to obtain a good quality but low cost pump laser. With introduction of higher power laser diodes this system is not a cost effective source for pumping LiSAF.

\section{New Diode based Solid State Pump Source}

In an attempt to overcome some of the cost / performance issues associated with the pump module, LIGHTWAVE has pursued an aggressive effort to develop a new source for pumping Cr:LiSAF. Under this contract we have designed and developed a YAG based frequency doubled pump source at $660 \mathrm{~nm}$. Much of the design work for this laser has been shared with our newly developed and recently introduced $532 \mathrm{~nm}$ laser (model 240).

The powerful new Series 240 lasers depicted in Figures 10 and 11 are CW, diode -pumped frequency-doubled Nd:YAG lasers and are excellent sources for pumping Cr:LISAF or Ti:Al2 3 . The Series 240 lasers can be used in a variety of other applications such as spectroscopy, medical, and reprographics. These lasers employ the new Direct-

Coupled Pump (DCP) technology that directly couples pump light from a diode array into the laser crystal to produce a compact and reliable laser. The Series 240 Lasers are an 
attractive alternative to ion lasers for many applications. The system includies a laser head consisting of the optical pump and resonator components, a power supply that includes a user interface with numerous features and diagnostics, and a closed-loop chiller for removing heat from the laser head. These lasers do not require special utilities such as a 3 phase $220 \mathrm{~V}$ supply line. The Series 240 lasers provide the advantages of a diode-pumped system-reliability, compactness, and efficiency.
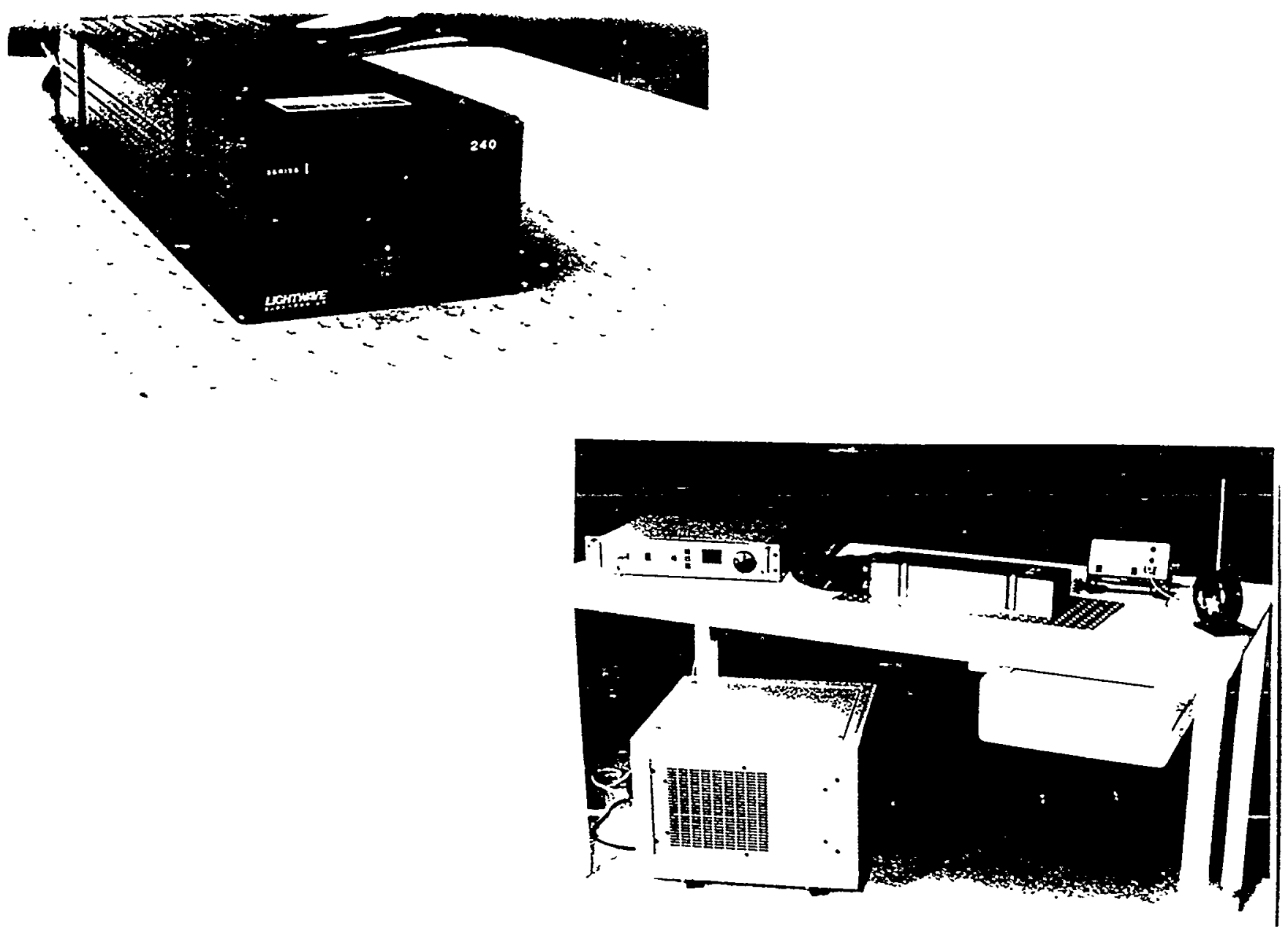

Figures 10,11

In addition to providing a diffraction limited beam $\left(\mathrm{M}^{2}\right.$ of less than 1.2$)$, both models provide very stable, low noise output. Amplitude stability for our model $240532 \mathrm{~nm}$ laser has been rated to be less than $.5 \%$ from $3 \mathrm{~Hz}$ to $3 \mathrm{MHz}$ with a linearly polarized beam and a polarization ratio of greater than $1(0): 1$. This laser has been rated for an output power of 2 
$\mathrm{W}$ and provides a full angle beam divergence of $.4 \mathrm{mrad}$. The system can operate at ambient temperatures between 15 and $35^{\circ} \mathrm{C}$, but may be stored in a zero to $50^{\circ} \mathrm{C}$ environment. The Series 240 lasers are compact and relatively light weight. The laser head itself is $11 \times 39 \times 47 \mathrm{~cm}$ and weighs only $9 \mathrm{~kg}$. Since our introduction of the model $240 \mathrm{at}$ CLEO-95, the response has been overwhelming and manufacturing production of this laser has already begun.

The model $240-\mathrm{R}$ provides $1 \mathrm{~W}$ of $\mathrm{CW}$ radiation at $660 \mathrm{~nm}$ with amplitude noise "tability similar to the 240 . This laser is a poten" $1:$ ! replatcement for the less cost effective, bulky, and high maintenance Krypton ion systems. Due to a number of technical challenges, however, the model $240-\mathrm{R}$ is still in its development stage. Although we are

generally pleased with the results of construction of a $240-R$ prototype; full production of this system requires further design work. In the next section some design challenges as well as laser characteristics for the model $240-\mathrm{R}$ are discussed.

\section{a) System Design and Optical Challenges}

The goals of optical design were to provide a stable and low noise source of light at $660 \mathrm{~nm}$. The laser was designed to run CW and have a diffraction limited output beam. Figure 12 shows a schematic of a basic cavity used for our purposes. This cavity is widely known as a $Z$ cavity. Infrared light emitted by the active medium is trapped bythe highly reflective mirrors and since all mirrors have low loss at the fundamental frequency, a very high intensity of IR light builds up in the cavity. Introduction of a nonlinear medium to this cavity, can then convert some of the intracavity light into its second hamonic. Since frequency conversion takes place inside the cavity, it is important to design at least one mirror to have a high loss at the newly generated wavelength. Overall, this scheme has worked well for us and we have been able to extract multiwatt levels of frequency doubled light from them. However, through the course of construction of a model 240-R prototype we were faced with many optical difficulties. These included fundamental wavelength 


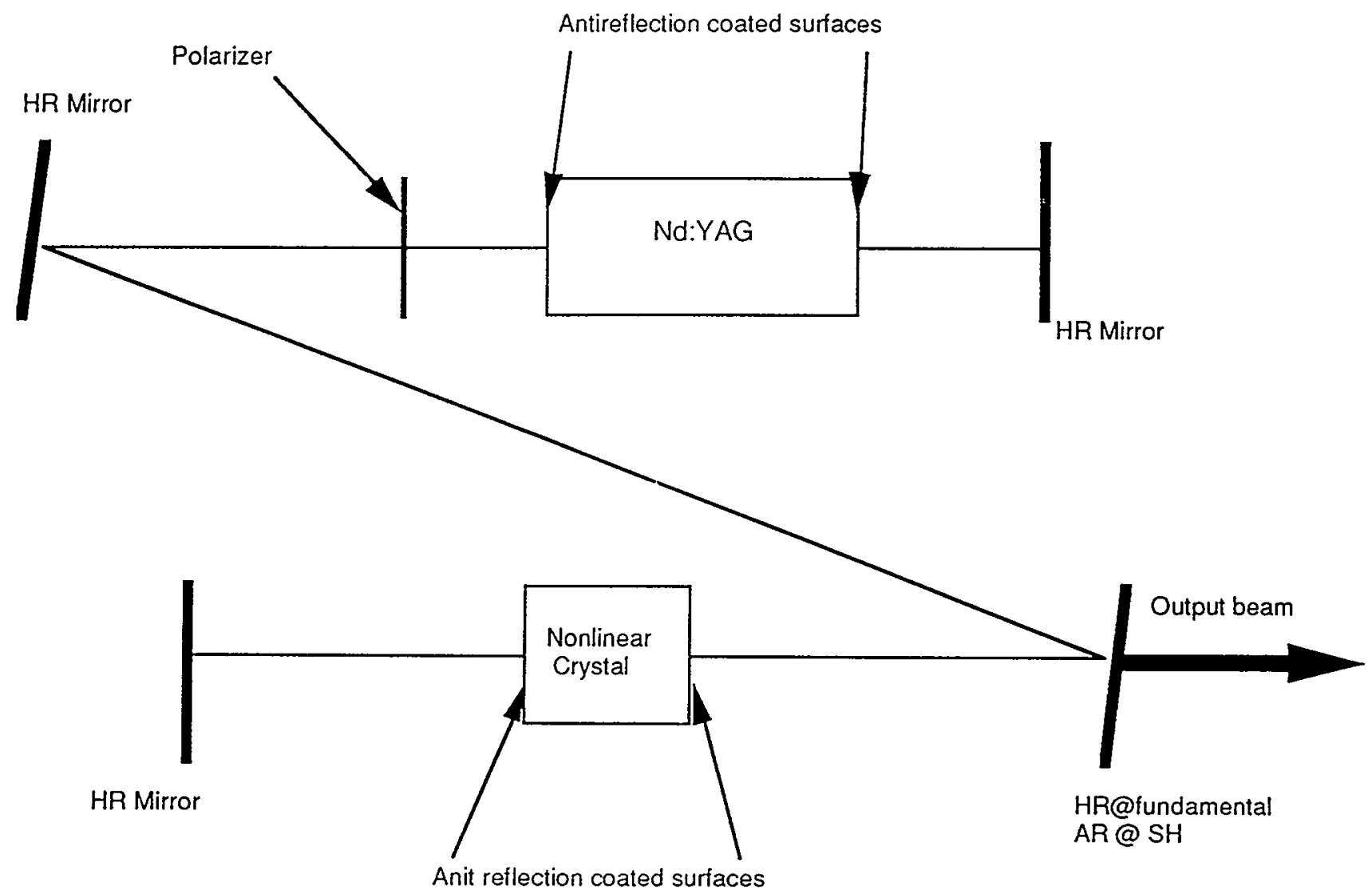

Figure 12

stability, SHG medium characteristics and requirements, as well as crystal and mirror coatings.

In this project we used the $1.319 \mu \mathrm{m}$ line of the Nd:YAG as our fundamental wavelength. We then frequency double this light into the visible. Unfortunately, the Nd:YAG also emits radiation at $1.338 \mu \mathrm{m}$ with comparable gain. Since mirror and coating reflectivities are broadband, lasing action takes place at both lines. A laser, by definition, runs at its lowest loss state. If we were to ignore the undesired wavelength within our ravity, and try to frequency double the light, we would obtain very little SHG light (few milliwatts). Since introduction of the nonlinear medium to the cavity would raise the loss at 
one wavelength, the amount of infrared light at the other wavelength would increase considerably and efficient frequency conversion would not take place. One way to eliminate this problem is by introduction of a thin etaion into the cavity. An etalon of right thickness and surface reflectivity can discriminate between the two wavelengths and allow only one wavelength to be present. Another way to overcome the dual line difficulty is to place a birefringent filter in the cavity. In either case, new losses are introduced to the cavity which result in reduced overall intracavity fundamental power..

Currently there are several nonlinear materials which can be used to frequency double $1.319 \mu \mathrm{m}$ light. In this project we considered one biaxial crystals, one uniaxial crystal and a periodically poled material. The difficulties associated with each of the above are discussed below.

One material we considered was $\mathrm{LiB}_{3} \mathrm{O}_{5}$ (Lithium Triborate, also known as LBO). This material has a relatively high damage threshold, and can non criticall; phase match our fundamental wavelength at room temperature. LBO is a biaxial crystal and although we have never seen any evidence of this, it is considered to be slightly hygroscopic. Since the crystal is to work inside the cavity, it is imperative to place anti reflection coating on its optically active surfaces. One problem associated with this material is its thermal expansion. In our case, the fundamental light was numped along the $\mathrm{x}$ axis of the crystal. This makes the optically active surfaces in the $y_{1}$.ane of the crystal. The coefficient of ther.: expansion of LBO along $y$ and $z$ are $-8.8 \times 1000-5 / K$ and $3.4 \times 10-5 / K$ respei, $: y$. Thermal variations can place major strain on surface coatings. Major degradation of anti reflection coating on LBO surfaces result in lower intracavity fundamental power. Also this unusual thermal expansion property can produce mechanical difficulties as well. Any excess heat generated in the crystal must be extracted from the material to maintain a constant temperature and hence provide a low noise system. Crystal temperature control can be achieved by placement of the LBO in a highly thermally conductive housing such as aluminum or copper. Thermal contact must be made between 
the housing walls and the crystal. As the temperature in the LBO is changed and the crystal dimensions are slightly altered and thermal contacts weakened. In the case of thermal expansion, extraneous pressure could build up in the crystal causing it to crack.

We also considered a relatively well known uniaxial crystal. $\mathrm{LiNbO}_{3}$ (Lithium Niobate) is a widely available, low cost material with a high nonlinear coefficient. This material does not display any unusual thermal characteristics and placement of anti reflection coating on its surfaces has been relatively well characterized. In addition, $\mathrm{LiNbO}_{3}$ is not considered hygroscopic. A usual problem associated with this material however is that at temperatures below $100^{\circ} \mathrm{C}$ photorefractive damage is quite probable. In our case phase matching temperature was $365^{\circ} \mathrm{C}$, well beyond this damage region. Such a high temperature however introduces a number of other problems. One of the most important issues associated with taking the crystal to $365^{\circ} \mathrm{C}$ is that as the crystal is heated electrostatic charges build up on its surfaces. If adequate care is not taken, a sudden discharge could take place, destroying the crystal. Also at high temperatures performance of anti reflection coatings may be reduced considerably. A third problem is maintaining a uniform temperature profile within the nonlinear material. Since the ambient temperature is much lower than the phase matching temperature, it is possible to introduce strong thermal gradients within the crystal. Strong gradients can not only reduce conversion efficiency, but can also destroy the crystal altogether. Yet another issue is outgassing of the material at high temperarures. In order to ensure a long lifetime period for $\mathrm{LiNbO}$ : irystal needs to be in an oxygen rich environment. This in itself is a major problem si:: oxygen is highly reactive, and can accelerate surface and material degradation and cause oxidation of materials in and around the nonlinear crystal.

A third material we considered for this project was Periodically Poled Lithium Niobate (PPLN). A periodically poled nonlinear device may provide much higher overal nonlinearities and hence a better frequency conversion efficiency. The effective nonlinear coefficient ( $\mathrm{d}_{\mathrm{eff}}$ ) for a $\mathrm{LiNbO}_{3}$ crystal to frequency double our fundamental is $4.3 \mathrm{pm} /$ Volt. 
In contrast deff for PPLN is about $17 \mathrm{pm} /$ Volt. Since the intensity of the second harmonic light is dependent on the square of the effective nonlinearity, keeping all other parameters constant, PPLN provides about 15 times more conversion than the conventional method. Many practical problems however exist in construction of suitable PPLN.

The preferred technique for fabricating PPLN is called electric-field poling. Over the past year, LIGHTWAVE Electronics has developed the in-house capability to fabricate PPLN. We initially followed the recipe developed at Stanford University. This recipe inyolves designing a lithographic mask to produc? ? desired electrode pattern. Then, using standard lithium niobate wafers ( 3 " diameter x $0.5-1.0 \mathrm{~mm}$ thick), photolithography and metallization steps are carried out to yield the structure shown in Fig. 13. The mask fabrication is done at Photo Sciences, Torrance, CA, and the photolithography is done at one of several vendors. Both the mask fabrication and the photolithography are very standard procedures, and are considered to be "easy-to-do" by the electronics industry.

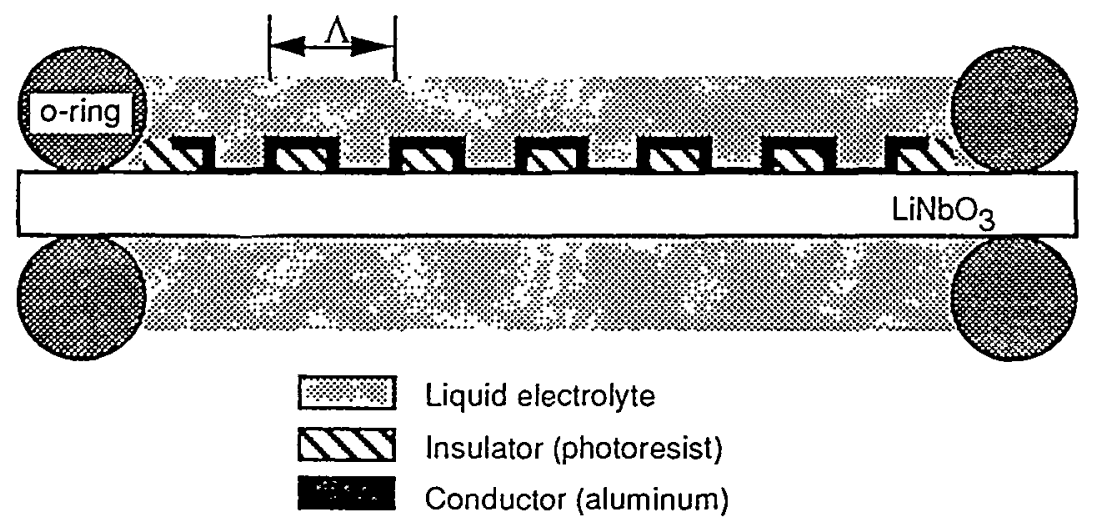

Figure 13

Fig. 13. Schematic of the standard electrode structure (metal over photoresist) used to produce periodically-poled lithium niobate (PPLN). The whitc area is a lithium niobate wafer, the striped area is photoresist $(2 \mu \mathrm{m}$ thick), and the black area is aluminum $(0.2 \mu \mathrm{m}$ thick). Electrical contact is made by covering both surfaces with an electrolyte $(\mathrm{LiCl})$ (gray area). The patterning in the photoresist is done lithographically; its spacing $(\Lambda=26-32 \mu \mathrm{m})$ determines the output wavelength of the OPO.

The processed lithium niobate wafers are poled at LIGHTWAVE Electronics by placing them between electrodes. Electrical contact is made by sandwiching the wafer 
between two o-rings held by plexiglas blocks. A liquid electrolyte $(\mathrm{LiCl})$ is inserted inside the o-rings so it covers both surfaces. Wires from the high voltage source which deliver 12 - $24 \mathrm{kV}$ pulses of $\sim 100$ second duration are dipped into the $\mathrm{LiCl}$. The exact voltage and pulse duration depends on the thickness and size of the poled sample. After poling, the photoresist and aluminum are stripped off, and wafer is immersed in HF acid. HF acid differentially etches the $+z$ and $-z$ surfaces, producing a step-like pattern on the wafer surface which allows visualization of the ferroelectric domain pattern. The pattern is visible Wutu itc naked eye, but can more easily be obstr red with a w.....uscope. If the domain pattern is judged to be of sufficient quality, the ends of the poled lithium niobate crystal are polished and coated to yield a finished crystal as shown in Fig. 14.

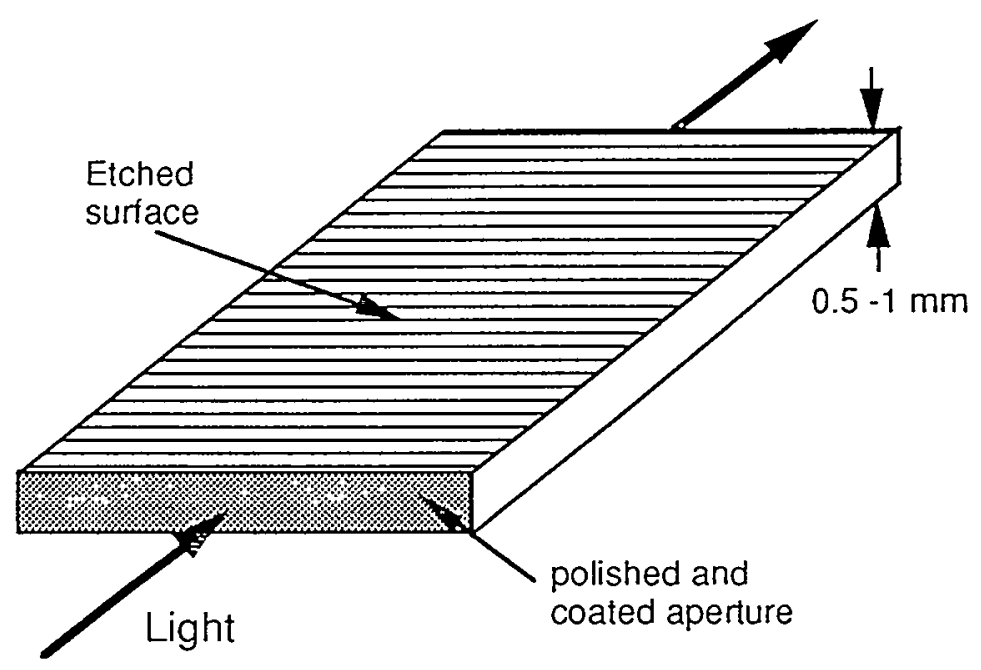

Fig. 14 Schematic of a finished PPLN crystal (not drawn to scale). The crystal is $0.5-1 \mathrm{~mm}$ thick, and can be up to $70 \mathrm{~mm}$ long. The top surface was etched in HF acid to reveal the ferroelectric domain pattern dictated by the lithographic mask. The aperture for the light is determined by the wafer thickness.

Another issue we faced in development of a $660 \mathrm{~nm}$ pump source was degradation of optical coatings. These degradations were observed both on reflective and anti reflective surfaces. As mentioned above, thermal effects in the nonlinear material can cause surface - tension to damage the coatings In the case of LBO we observed a peeling effect with certain coating materials after a few days of operation. $\mathrm{LiNbO}_{3}$ coating behaved much 
better and did not seem to be effected by the high crystal temperature. We also observed major damage to reflective suriaces of the mirrors. If the intracavity surface intensity is too high (more than 100 megawatts pur square $\mathrm{cm}$ ), then the reflective coating on the mirrors can burn. In several instances we observed a chain reaction effect where the coating in one spot had burned and as we had attempted to change the location of the beam on the mirror, it had etched a trace of its path creating burn lines. To overcome this problem it is possible to reduce mirror surface intensity by decreasing the diode pump powers. This process hovever also reduces the amount of intrauvity light and henc. the overall output performance of the laser and is not a feasible option. The best way is to maintain large mode sizes on reflective surfaces Another issue that can reduce mirror reflectivity is that it is possible for mirror coating materials to absorb water from the air over time. In this case degradation is slow and occurs over a period of months. Since there is a slight absorption of the fundamental light by water, this can have a severe effect on overal laser performance. A good thing to do in such a case is to make sure that the mirrors are being kept in a relatively dry environment.

\section{b) Mechanical Mounting}

Permanent placement of optical components in the laser head is one of the cruicial aspects in designing a reliable and robust product. Mechanical mounts and housings can effect laser stablility and noise properties considerably. In designing a good housing mount for the nonlinear crystal, several issues had to be taken into account. In the case of frequency doubling with $\mathrm{LBO}$, type II phase matching takes place at room temperature. An interesting phenomenon associated with room temperature phase matching is that if the crystal temperature is not controled well, the laser output power displays oscilatory behavior. When maximum conversion is taking place the crystal undergoes self-heating and its temperature increases. This in turn takes the crystal out of phase matching conditions and effects conversion which reduces heating of the crystal. The material cools 
down, phase matching becomes possible, and the crystal starts to heat up once again. This problem can be overcome by the use of a well designed crystal housing which extracts extra heat away and helps maintain a stable temperature with minimal gradiants through the crystal.

Figure 15 shows a drawing of the housing oven which we used. As depicted the crystal housing sits on a TEC cooler for accurate temperature control. To obtain a good thermal flow from the other side of the TEC to a heat sink (the laser head), we needed a design that would allow good thermal conductivity and angular adjustments.

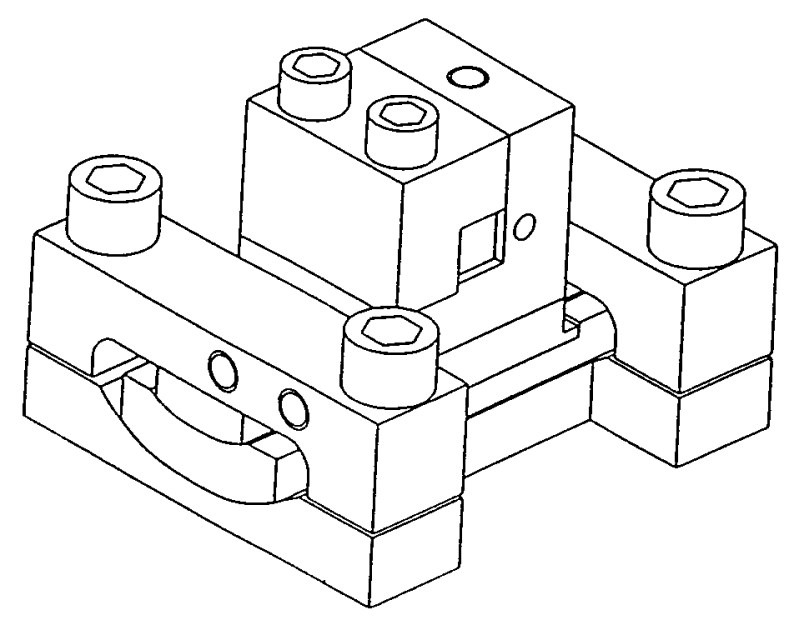

Figure 15

We acheive this by designing a rotaional locking mount which would not only allow large thermal gradients, but also would lock the position of the crystal permanently after all optical components of the cavity had been positioned. 


\section{c) Electronics and Control Module}

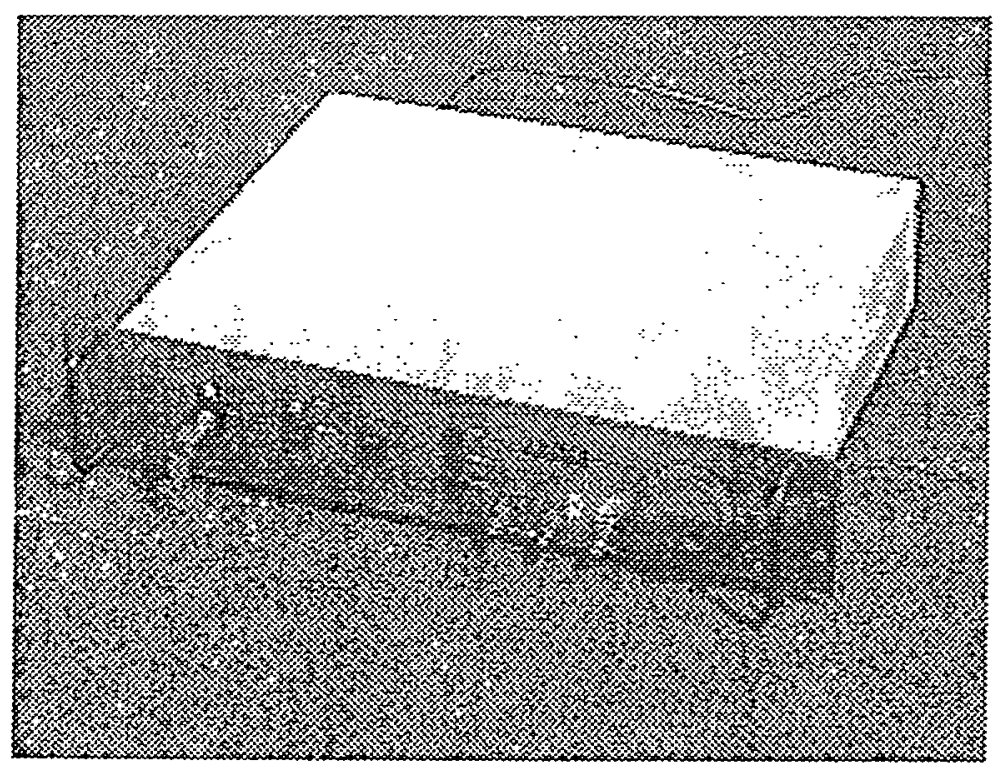

Figure 16

Figure 16 shows the power supply's front panel. With the laser head set up and all connections properly made, the laser can be controlled entirely from the front panel of the power supply. This system has many useful features and the two line display offers the user with system diagnostics. Among some of the features are diode current and temperature settings, diode temperature sensing, monitoring laser power, and display showing tota: hours of operation.

The current adjustment feature allows the operator to view the diode current in amps. The control dial set on the front panel may be used to change the current level below the maximum level. Diode current changes are made in 0.15 amp increments. To save this value the user can then press a set button. Of course output power, stability, beam divergence, etc., change as the diode current is decreased. The display on the front panel shows DC current of the laser diode. The power supply's current circuitry uses two 
current monitors. The monitors are used for overcurrent fault and short circuit protection. Their values are available to the microprocessor for checking and display.

The temperature at which the diodes operate determines the wavelength of the diodes. Thus, to ensure optimal absorption into the Nd:YAG, the diode temperature needs to be properly tuned. For various scientific applications, the user might need to alter this setting. Although diode temperature setting is done here at LIGHTWAVE, the value may be altered by the user by adjusting the temperature set point with the control dial.

1 Ii de temperature sensor monitors the tem $m_{p}$ rature of th ......es and then sends this information to temperature control electronics as well as the control module. This feature is used to monitor the temperature of the diodes at the reference location in the laser head. The value of the temperature sensor normaly agrees within $1^{\circ} \mathrm{C}$ of the set point, except in transient period following a change in the set point, or after turn on. A typical transient period for our model 240 is about 20 minutes, as the reservoire water temperature adjusts to its set point.

A very useful feature is the display of total operation time. The hours of operation display represents the total number of hours the laser dic des have run. This feature is a very useful tool for troubleshooting the system in case of service. It also alows the user to know how long the laser has been running and can provide an operational timeli 2.

\section{Conclusion and Future "tions}

In this project we have demonsi... $s$ d that LiSAF is a usefui material for producing electrons at CEBAF. There are however many practical difficulties associated with this materials upper state lifetime quenching at room temperature. Damage threshold also seems to be relatively low (especially for doping levels above 1\%). For many applications Cr:LiSAF may prove not to be a practical choice of material. Although femto second pulses have been generated in the past using this material, obtaining average power of hundreds of milliwatts is quite a challenge. While $\mathrm{Ti}_{1} \mathrm{Al}_{2} \mathrm{O}_{3}$ can demostrate roughly the 
same modelocked performance, it has the advantage of higher damage threshold and does not undergo upper state lifetime quenching at room temperature.

The response to Lightwave Electronics recent introduction of a 2 Watt $532 \mathrm{~nm}$ (LIGHTWAVE laser model 240) has been overwhelmingly positive. The technology developed in phase II of this project enables Lightwave Electronics to introduce a new product to model 240 production series. This laser would be a potential replacement for the less reliable, bulky and less efficient krypton ion lasers. For several years Lightwave Electronics has provided a modelocked laser 'model 131) using bot. $V \AA G$ and YLF based crystals. As a result of this contract, we are now capable to incorporate the new LiSAF technology into production lines although no manufacturing plans have been drawn for such a project yet.

We are now able to produce four different products (diode pump module, the 2 watt $532 \mathrm{~nm}$ system, a modelocked $100 \mathrm{MHz}$ LiSAF system, and a 1 watt $660 \mathrm{~nm}$ laser), all either partially or fully developed under this contract. Depending on the needs of a particular application these lasers can provide more flexibility and accommodate the needs of many scientific and industrial users, and provide more robust, cost effective and energy efficient environmentally sound systems. 


\section{References}

1. J.S. Fraser and R.L. Sheffield, "High-brightness injectors for RF-driven freeelectron lasers," IEEE J. Quantum Electron., Vol. QE-23, pp. 1489-1496, 1987

2. Advanced Accelerator Concepts, Frederick E. Mills, Ed., American Institute of Physics, New York, 1987

3. J.M. Dawson, "Plasma particle accelerators,", Scientific American, pp. 54-62, March 1989

4. K.J. Weingarten, M.J.W. Rodwell, and D.M. Bloom, "Picosecond optical sampling of GaAs integrated circuits," IEEE J. Quantum Electron., Vol. QE-24, pp. 198-220, 1988 